"This is the pre-peer reviewed version of the following article: [FULL CITE], which has been published in final form at http://onlinelibrary. wiley.com/doi/10.1002/2015GL065036/full. This article may be used for non-commercial purposes in accordance with Wiley Terms and Conditions for Self-Archiving.

\title{
Dissolution Patterns and Mixing Dynamics in Unstable Reactive Flow
}

Juan J. Hidalgo, ${ }^{1,2}$ Marco Dentz, ${ }^{1,2}$ Yoar Cabeza, ${ }^{1,2,3}$ and Jesus Carrera ${ }^{1,2}$

Corresponding author: J. J. Hidalgo, IDAEA-CSIC, Jordi Girona 19, Barcelona, 08034, Spain. (juanj.hidalgo@idaea.csic.es)

${ }^{1}$ Spanish National Research Council

(IDAEA-CSIC), Barcelona, Spain.

${ }^{2}$ Associated Unit: Hydrogeology Group

(UPC-CSIC)

${ }^{3}$ Universitat Politècnica de Catalunya

(UPC), Barcelona, Spain. 
We study the fundamental problem of mixing and chemical reactions under a Rayleigh-Taylor-type hydrodynamic instability in a miscible two fluid system. The dense fluid mixture, which is generated at the fluid-fluid interface, leads to the onset of a convective fingering instability and triggers a fast chemical dissolution reaction. Contrary to intuition, the dissolution pattern does not map out the finger geometry. Instead, it displays a dome-like, hierarchical structure that follows the path of the ascending fluid interface and the regions of maximum mixing. These mixing and reaction hot spots coincide with the flow stagnation points, at which the interfacial mixing layer is compressed and deformed. We show that the deformation of the boundary layer around the stagnation points controls the evolution of the global scalar dissipation and reaction rates and shapes the structure of the reacted zones. The persistent compression of the mixing layer explains the independence of the mixing rate from the Rayleigh number when convection dominates. 


\section{Introduction}

Unstable fluid systems often experience chemical reactions coupled to the mixing driven by flow instabilities. For example, mantle convection determines the distribution of minerals in the Earth crust [Kouchi and Sunagawa, 1983; Tackley, 2000; Couch et al., 2001]; Rayleigh-Taylor instabilities speed up burning processes in supernovae explosions [Schmidt, 2006]; and flow fluctuations are key for the performance of microfluidics reactors [deMello, 2006]. Correspondingly, chemical reactions may trigger (or suppress) fluid instabilities by changing the density of the fluids [Eckert and Grahn, 1999; Almarcha et al., 2010; Andres and Cardoso, 2011; Loodts et al., 2014; Cardoso and Andres, 2014]. In porous media, reactions can alter the solid matrix and create persistent porosity structures that, in turn, transform the flow regime [Steefel and Lasaga, 1990; Golfier et al., 2002; Szymczak and Ladd, 2009, 2011; Ritchie and Pritchard, 2011; Szymczak and Ladd, 2013]. This is a relevant process in karst formation [Gabrovšek and Dreybrodt, 2010], petroleum reservoirs [Fredd and Fogler, 1998], and the migration and dissolution of $\mathrm{CO}_{2}$ in saline aquifers [Lindeberg and Wessel-Berg, 1997; Verdon and Woods, 2007; Backhaus et al., 2011; Hidalgo et al., 2012; Hewitt et al., 2013]. The relation between unstable flow patterns, persistent flow structures, and coupled mixing and reactions is central to the understanding of natural phenomena and engineered systems from the nano to the kilometer scale [Tél et al., 2005].

We focus here on a mixing-limited dissolution reaction in a system subject to a RayleighTaylor instability. Mixing-limited reactions are fast compared to the mass transfer time scales so that the system can be considered locally at chemical equilibrium [Kechagia 
et al., 2002], a situation ubiquitously encountered in nature. The reaction rate is then proportional to the mixing rate [De Simoni et al., 2005; Sanchez-Vila et al., 2007] and flow instabilities control the reactions and the location and distribution of matrix alterations. We analyze the relation of the reaction and mixing rates with the unstable flow structure formed by stagnation points along the interfacial boundary layer of the Rayleigh-Taylor instability.

\section{Governing Equations}

We consider a miscible two fluid system characterized by a non-monotonic densityconcentration curve such that the mixture is denser than either pure fluid [Neufeld et al., 2010; Hidalgo et al., 2012]. Initially, the fluid interface is stable with the light fluid laying on top of the denser fluid. Upon mixing, the interface quickly destabilizes due to instability created by density increases arising from the fluid mixture. The fluids are in chemical equilibrium with the porous matrix but not with each other so that mixing perturbs the equilibrium and triggers a chemical reaction.

\subsection{Chemical system}

The system is subject to an instantaneous dissolution reaction in which the concentrations of the aqueous species A and B follow the relation [Appelo and Postma, 2005; Sup, a]

$$
c^{\AA}=C_{\mathrm{B}},
$$

where $c$ denotes a dimensionless concentration scaled with ${ }^{\sqrt{ }} K / 2$, with $K$ the chemical equilibrium constant. In the specific case of the dissolution of calcite, the cubic 
relation (1) arises for the concentration of calcium ions (A) and carbon dioxide partial pressure (B) [Appelo and Postma, 2005; Sup, a].

The species concentrations $c_{\mathrm{A}}$ and $c_{\mathrm{B}}$ define the conservative component $u=c_{\mathrm{A}}+C_{\mathrm{B}}$, whose reaction rate is zero [Sup, a]. The component $u$ is conserved under transport and reaction. Being a conservative quantity, it can be expressed in terms of the mixing ratio of the compositions of the top $(T)$ and bottom $(B)$ fluids as $U=\alpha U_{T}+(1-\alpha) U_{B}$. Note than only conservative quantities can be expressed in terms of the mixing ratio $\alpha$.

\subsection{Flow and transport}

Assuming that both fluids are incompressible, and using the Boussinesq approximation, the governing equations for variable-density single-phase flow in a bidimensional porous medium take the following dimensionless form [Riaz et al., 2006; Hidalgo et al., 2013]:

$$
\begin{aligned}
& \nabla \cdot \mathbf{q}=0 \\
& \mathbf{q}=-k(\nabla p-\rho \hat{\mathbf{z}}) \\
& \frac{\partial \alpha}{\partial t}+\frac{1}{\varphi} \mathbf{q} \cdot \nabla \alpha-\frac{1}{\mathrm{Ra}} \nabla^{2} \alpha=0 .
\end{aligned}
$$

Equation (2) expresses the mass conservation for an incompressible fluid, (3) is the Darcy equation, and the mixing ratio $\alpha$ follows the conservative advection-diffusion equation (4).

These equations are non-dimensionalized by using the following characterstic scales. The coordinates $x$ and $z$ are scaled with respect to the initial height of the interface $H_{0}$ measured from the bottom of the box with $\hat{\mathbf{z}}$ the unit vector in the direction of gravity. Permeability $k$ and porosity $\varphi$ are referred to the initial homogeneous values $k_{0}, \varphi_{0}$. Pressure $p$ is measured with respect to a hydrostatic datum. Darcy velocity $\mathbf{q}$ is scaled by the characteristic velocity $q_{0}=k_{0} \Delta \rho_{5} g / \mu_{0}$, where $\Delta \rho_{m}$ is the difference between 
the maximum density and the initial density of the bottom fluid, $g$ is the gravitational acceleration, and $\mu_{0}$, the dynamic viscosity, is considered constant. The dimensionless density difference with respect to the bottom fluid is denoted by $\rho$. The contribution of the dissolved matrix is neglected so that $\rho$ depends only on the mixing ratio. $\rho$ takes a value of 0 at $\alpha=0$, increases to a maximum value of 1 at $\alpha=\alpha_{m}$, where $m$ denotes maximum, and decreases to a negative value at $\alpha=1$ [Sup, a]. Finally, time $t$ is expressed in terms of the advective time $t_{\mathrm{adv}}=\varphi_{0} H_{0} / q_{0}$ and the Rayleigh number Ra is defined as

$$
\mathrm{Ra}=\frac{q_{0} H_{0}}{\varphi_{0} D_{m}}
$$

where $D_{m}$ is the diffusion coefficient. We assume that all species have the same diffusion coefficient to avoid violating the charge balance [Lichtner, 1985].

\subsection{Reaction rate}

The reaction rate for a mixing limited reaction is given by [De Simoni et al., 2005; Sup,

a]

$$
r_{A}=\varphi \frac{d^{2} c_{\mathrm{A}}}{d \alpha^{2}} X, \quad X=\frac{1}{\mathrm{Ra}}|\nabla \alpha|^{2},
$$

where the factor $\frac{d^{2} c_{A}}{d \alpha^{2}}$ can be computed analytically using the definition of $u$ and (1), and $X$ is the scalar dissipation rate, whose role for the quantification of mixing has been discussed elsewhere [Le Borgne et al., 2010; Hidalgo et al., 2013]. The expression for $r_{A}$ reveals the control of mixing over reactions.

Our model is closed by accounting for the porosity changes caused by the dissolution of the porous matrix. The rate of change of porosity is proportional to the reaction rate 
and given by [Sup, a]

$$
r_{\varphi} \equiv \frac{1 d \varphi}{\varphi d t}=-\frac{\mathrm{N}_{\varphi} d^{2} c_{\mathrm{A}}}{\operatorname{Ra} d d^{2}}|\nabla \alpha|^{2},
$$

where $\mathrm{N}_{\varphi}={ }^{\sqrt{ }} K T\left(2 V_{m}\right)$, with $V_{m}$ the molar volume of the solid phase. In the following, we refer to (7) as the dissolution rate.

Permeability $k$ depends on $\varphi$ through a Kozeny-Carman law $k=\varphi^{3}$ in dimensionless form [Bear, 1972; Sup, a]. The cubic dependence produces small and smooth variations of permeability making flow, transport and reaction to be weakly coupled.

\section{Mixing and Dissolution Patterns}

The coupled unstable reactive flow and transport problem (2)-(4)-(7) is solved in a rectangular domain $(x, z) \in[0,1] \times[0,2]$ with periodic boundary conditions in $x$ and no flow boundaries at the top and bottom. The equations are discretized in space using 2nd-order finite volumes and 6th-order compact finite differences (4th-order for boundary conditions). The system is propagated in time using an explicit 3rd-order Runge-Kutta scheme [Hidalgo et al., 2013].

The results of a typical simulation are shown in Fig. 1 at a time $(t=15)$ when convective instabilities are well developed [Fig. 1 (a)]. The fluids mix by two mechanisms: the flux through the interface and the diffusion across the finger boundaries. However, reactions cluster at the fluid interface where there is a boundary layer created by the competition between diffusion and the compression caused by the upwelling less dense fluid displaced by the fingers. Unexpectedly, reactions along the fingers fringe are rather weak [Fig. 1 (b, c, e)]. No significant scalar gradients nor dissolution is observed because the concentration contrast is attenuated by diffusion. The resulting dissolution pattern resembles a series 
of intertwined dome-like structures that follow the ascending path of the highly reactive compressed fluid interface [Fig. 1 (d, f)].

The fluid interface is compressed and stretched at the flow stagnation points at a stretching and compression rates $\gamma=\partial q_{x} / \partial x=-\partial q_{z} / \partial z$. This forms hot spots, i.e., regions where mixing and reaction are maximum [Agrawal et al., 2007; Gérard et al., 2012], which follow the strain rate distribution [Fig. $2(\mathrm{a}, \mathrm{b}, \mathrm{c})$ ]. The hot spots appear on either side of the stagnation points [Fig. 2 (d)] where the compression of the boundary layer is highest.

The control that the fluid structure exerts on mixing and dissolution is also reflected in the akin evolution of the global dissolution and scalar dissipation rates defined by integration of (6) and (7) over the flow domain $\Omega$

$$
\begin{aligned}
& (X)=\frac{1}{\operatorname{Ra}}_{\Omega}^{r} d \Omega|\nabla \alpha|^{2}, \\
& \left(r_{\varphi}\right)=-{\frac{N_{\varphi}}{\operatorname{Ra}}{ }_{\Omega} d \Omega \frac{d^{2} c_{A}}{d \alpha^{2}}|\nabla \alpha|^{2} .}^{r} .
\end{aligned}
$$

They display three different regimes [Fig. 3] consistent with previous experimental observations [Backhaus et al., 2011; Slim et al., 2013]. At the beginning mixing and reaction are driven by diffusion across the interface and follow the characteristic $t^{-1 / 2}$ behavior After the time for the onset of convection, which scales as $\mathrm{Ra}^{-1}$ [Riaz et al., 2006], a convection-dominated regime develops. This regime is characterized by a sudden increase of both observables towards a plateau whose value is independent of Ra. At larger times, as the bottom fluid gets better mixed with the top fluid, the density contrast decreases and convection attenuates. During this convection shutdown regime the mixing and reaction rate decay quickly [Slim et al., 2013; Slim, 2014; Bolster, 2014]. 


\section{Interface Stretching Model}

In the three regimes, mixing and reaction are dominated by the processes at the fluids interface. Therefore, their evolution can be quantified by the dynamics of the hot spots created by the stagnation points.

The scalar transport in the vicinity of a stagnation point can be described by the advection-diffusion equation [Ranz, 1979]

$$
\frac{\partial \alpha}{\partial t}=V \tilde{z}_{\partial \tilde{z}}^{\partial \alpha}+\frac{1 \partial^{2} \alpha}{\operatorname{Ra} \partial \tilde{z}^{2}}
$$

where $\tilde{z}$ is the vertical position in the inertial system moving with the interface. The horizontal scalar gradients along the interface are small and disregarded here. The solution for $\alpha$ along its characteristics gives

$$
\alpha=\alpha_{b}+\frac{\Delta \alpha_{b}}{2} \operatorname{erfc} \underbrace{\backslash}_{\sqrt{ } \frac{\tilde{z}}{2 s^{2}}}
$$

where $\Delta a_{b}=1-\alpha_{b}$ is the difference between the mixing ratio above $(\alpha=1)$ and below $\left(\alpha=\alpha_{b}\right)$ the interface and $s$ is the thickness of the interfacial boundary layer. $s$ satisfies [Villermaux, 2012; Le Borgne et al., 2013]

$$
\frac{1 d s}{s d t}=-v+\frac{1}{\operatorname{Ra}^{2}}
$$

which expresses the competition between convective compression and diffusive expansion. At the scale of $s$, the flow velocity changes from the buoyant velocity $u_{b}$ of the upwelling fluid to the interface velocity at the stagnation point $u_{i}$ such that the compression rate is given by $\gamma=\left(u_{b}-u_{i}\right) / s$. The dimensionless buoyancy is given by $u_{b}=1-\rho\left(\alpha_{b}\right), u_{i}$ is proportional to the mass transfer rate across the interface. 
Using (11) in (8) and neglecting the contribution from the relatively diluted fingers below the interface we obtain the expression for $(X)$

$$
(x)=\sqrt{\sqrt{4 \pi}} \frac{\left(\Delta \alpha_{b}\right)^{2}}{\mathrm{sRa}}
$$

where $w_{e}$ denotes an effective interface length. Similarly, for $\left(r_{\varphi}\right)(9)$

$$
\left(r_{\varphi}\right)=-\frac{N_{\varphi} w_{e}\left(\Delta \alpha_{b}\right)^{2}}{\operatorname{sRa}} A_{r}, \quad A_{r}=\frac{d \tilde{z}^{d^{2} c_{\mathrm{A}} e^{-z^{2}}}}{d \hat{\alpha}^{2} 2 \pi},
$$

where $\hat{\alpha}=\alpha(\tilde{Z} s) . A_{r}$ depends on the shape of $\Delta \alpha_{b}$ across the interface and the details of the chemical system.

We now investigate the behavior of mixing and reaction during the three observed regimes using the proposed interface model.

\subsection{Diffusive regime}

In the diffusive regime, the fluid stratification is stable and the interface thickness increases by diffusion only $(\gamma=0)$ such that from (12) $s=\left(s^{2}+2 t / R a\right)^{1 / 2}$ with $s_{0}$ the initial thickness. The mixing ratio below the interface is $\alpha_{b}=0 . w_{e}$ is the length of the flat interface and equal to the domain width $w$. The dimensionless width is always $w=1$. However, we prefer to write it explicitly for sake of clarity. Thus, we obtain

$$
\begin{aligned}
& (X)_{\text {diff }}=\frac{w}{\operatorname{Ra} 4 \pi} S_{2} \underline{\mathrm{Ra}}^{\frac{2 t}{\sqrt{ }-{ }_{1 / 2}}}
\end{aligned}
$$

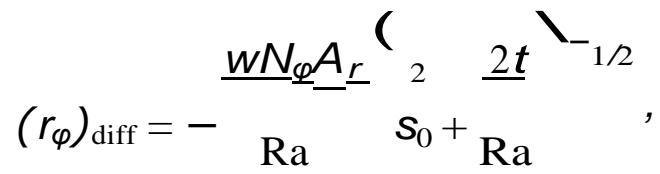

which match the early time behavior of the global dissolution and scalar dissipation rate [Fig. 3]. 


\subsection{Convective regime}

As fluids mix, the diffusive boundary layer at the interface becomes unstable, which leads to the characteristic fingering pattern illustrated in Fig. 1. The less dense fluid displaced by the fingers flows upwards towards the interface and creates a chain of stagnation points [Neufeldetal., 2010].

The upwelling fluid has an average concentration of $\alpha_{b}=0$ below the interface such that $u_{b}=1$. The interface velocity $u_{i}$ can be estimated from conservation of $\alpha$ given by $h_{0} w=m_{b}+\left(2 h_{0}-h\right) w$, where $h_{0}=1$ and $h$ are the dimensionless initial and current average heights of the interface, and $m_{b}$ is obtained by integration of $\alpha$ below the interface. Therefore, the average interface velocity $u_{i}=d h / d t=\left(d m_{b} / d t\right) / w$. We obtain $d m_{b} / d t$ by integrating (4) below the interface $\Gamma$, which yields

$$
\overline{u_{i}}=\frac{1}{w R a}_{\Gamma}^{r} d \Gamma_{\Gamma \tilde{z}}^{\underline{\partial \alpha}} \underset{w \operatorname{Ra} s}{\underline{w_{e}}} \underline{1 \Delta \alpha_{\underline{b}}},
$$

where we approximate the scalar gradient across the interface by the change in the mixing ratio over the interface thickness. We note that the dominant contribution to the integral originates from the region of width $w_{e}$ surrounding the stagnation points (see Figure $2 \mathrm{~d}$ ). Therefore, the typical interfacial velocity at the stagnation point given in terms of the average velocity is $u_{i}=u_{i} w / w_{e}$. Taking $\Delta \alpha_{b}=1$ for this regime because $\alpha_{b} \approx 0$ we have $V=1 / s-w /\left(s^{2} \mathrm{Ra}\right)$. We obtain self-consistently from the interface evolution (12) that the interface attains a steady state thickness $s_{B}=2 / \mathrm{Ra}$ at the time scale $1 / \mathrm{Ra}$, which sets the Batchelor scale for the convective regime [Batchelor, 1959].

The extension of a hot spot around a stagnation point is of the order of $s_{B}$. Thus, the effective width is $w_{e} \sim n_{f} s_{B}$, where $n_{f}$ is the number of fingers and is related to $s_{B}$ 
through the critical wavelength $\lambda_{c}$. Following Riaz et al. [2006], $n_{f}=1 / \lambda_{c}=\left(\beta_{c} \mathrm{Ra}\right) /(2 \pi)$ such that $n_{f} s_{B}=\beta_{c} / \pi$. We obtain $\beta_{c}=0.018$ from the average $n_{f}$ of the simulations. This value is $\alpha_{m}$ (here $\alpha_{m}=0.26$ ) times the one reported by Riaz et al. [2006] for a density law $\alpha_{m}=1$. The effect of $\alpha_{m}$ on the critical wavelength is also reflected on its observed influence on the average mixing during convection [Hidalgo et al., 2012]. Finally,

$$
\begin{gathered}
(X)_{\mathrm{conv}}=\frac{2}{\pi^{3 / 4}} \beta_{c} \\
\left(r_{\varphi}\right)_{\mathrm{conv}}={ }_{\pi}^{8} \beta_{c} N_{\varphi} A_{r},
\end{gathered}
$$

where we find $w_{e}=8 n_{f} s_{B}$ from $(X)$ in Fig. 3. The factor of 2 in (19) acknowledges that the extension of the reaction hot spots is twice that of mixing hot spots (see Figure 2 b, c). These expressions quantify the plateau values of both the global scalar dissipation and global dissolution rates as illustrated in Figure 3. Notably, both expressions are independent of the Rayleigh number.

\subsection{Convection shutdown regime}

As more solute mass is transferred across the interface, the density difference reduces and the stagnation points weaken [Hewitt et al., 2013]. The upwelling fluid velocity decreases as $u_{b}=1-\rho\left(\alpha_{b}\right)$, where $\alpha_{b}$ here is the average mixing ratio below the interface $\alpha_{b}=m_{b} /(h w)$, assuming complete mixing. We approximate [Sup, a] $u_{b} \approx\left(\Delta \alpha_{m} / \alpha_{m}\right)^{n}$, where $\Delta \alpha_{m}=$ $\alpha_{m}-\alpha_{b}$, and $n$ depends on the particular density law ( $n=2$ in this study). $u_{i}$ follows the same form as in the convective regime (17). Thus, $\gamma=\left(\Delta \alpha_{m} / \alpha_{m}\right)^{n} / s-\Delta \alpha_{b} /\left(s^{2} R a\right)$. The interface thickness $s$ evolves in a quasi-steady state manner because its relaxation time scale is much smaller than the one for the variation of the mixing ratio. Therefore, 
from (12) we obtain

$$
s=\frac{1+\Delta \alpha_{b}}{\Delta \alpha_{b}^{n} \mathrm{Ra}}
$$

where we approximate $\Delta \alpha_{m} \approx \alpha_{m} \Delta \alpha_{b}$ because $\alpha_{b} \ll 1$.

The evolution of $\Delta \alpha_{b}$ is obtained by deriving $m_{b}=\alpha_{b} h w$ with respect to time and using the global mass balance as closure [Sup, a]. Then

$$
\frac{d \Delta \alpha_{b}}{d t}=-\frac{w_{e} \Delta \alpha_{b}^{3+n}}{2 h_{0} W}
$$

We integrate this expression from the beginning of the shutdown regime at time $T_{S}$ to $t$ such that

$$
\Delta \alpha_{b}=1+\frac{(2+n) W_{e}}{2 h_{0} W}\left(t-T_{s}\right)^{I_{-1 /(2+n)}}
$$

Substitution of the latter in (13) and (14) gives

$$
\begin{gathered}
(X)_{\text {shutdown }}=\underline{\pi}_{\pi^{3 / 4} \beta_{c}} 1+\frac{(2+n) W}{2 h_{0} w} \underline{e}\left(t-T_{s}\right)^{I_{-1}} \\
\left(r_{\varphi}\right)_{\text {shutdown }}={ }_{\pi} \beta_{c} N_{\varphi} A_{r} 1+\frac{(2+n) w}{2 h_{0} W} \underline{e}\left(t-T_{s}\right)^{I_{-1}},
\end{gathered}
$$

where we used that $(X)_{\text {shutdown }},\left(r_{\varphi}\right)_{\text {shutdown }}$ are equal to their respective values in the convective regime at $t=T_{s}$. Note that $A_{r}$ evolves in time due to its dependence on $\Delta \alpha_{b}$. The results of simulations for $\mathrm{Ra} \in[2000,10000]$ show that $w_{e} \sim 0.002^{\sqrt{ }}$ Ra $[$ Fig. 3]. The fact that $w_{e}$ is inversely proportional to the transverse mass transfer is in agreement with a diffusive finger coarsening [Jenny et al., 2014].

\section{Conclusions}

In summary, our results show that mixing and mixing-limited reactions in an unstable 13

two fluid system are controlled by the evolution of stagnation points at the fluids interface. 
The porosity pattern caused by dissolution reflects the mixing history through the different regimes. It follows the path of the deformed interface, rather than the fingers as commonly presumed, and maps the regions of strongest mixing around the stagnation points. Global mixing and reaction rates are described by an interface mixing model that quantifies their evolution, and provides a physical explanation for their scalings. In particular, it explains the independence of mixing and dissolution from the Rayleigh number Ra in the convective regime.

In conclusion, reaction and mixing hot spots in unstable flow are associated to the flow structures that cause persistent fluid deformation. These findings have implications, for example, in the design of groundwater remediation and $\mathrm{CO}_{2}$ injection strategies, and the enhancement of mixing in microfluidic devices. The understanding of how the interaction between the flow instabilities and mixing dynamics shape the structure of reacted zones may shed new light on the interpretation of reaction patterns originated in unstable flow systems such as the ones found in igneous rocks formed during magma differentiation or in Karst formations due to carbonate dissolution

Acknowledgments. Data used for producing the figures can be obtained by solving the respective equations given in the manuscript. JJH and $\mathrm{MD}$ acknowledge the support of the European Research Council through the project MHetScale (FP7-IDEAS-ERC617511). YC acknowledges funding by the FP7 EU project TRUST (FP7-ENERGY309067). JC acknowledges funding by the Spanish Ministry of Economy and Competitiveness project MEDISTRAES (CGL2013-48869-C2-1-R). 


\section{References}

(a), see Supplemental Material at ... for the details of this derivation.

(b), see Supplemental Material at ... for simulation videos.

Agrawal, R., D. H. West, and V. Balakotaiah (2007), Modeling and analysis of local hot spot formation in down-flow adiabatic packed-bed reactors, Chem. Eng. Sci., 62 (18-20), 4926-4943, doi:10.1016/j.ces.2006.11.057, 19th International Symposium on Chemical Reaction Engineering - From Science to Innovative Engineering ISCRE-19.

Almarcha, C., P. M. J. Trevelyan, P. Grosfils, and A. De Wit (2010), Chemically driven hydrodynamic instabilities, Phys. Rev. Lett., 104 (4), doi: 10.1103/PhysRevLett.104.044501.

Andres, J. T. H., and S. S. S. Cardoso (2011), Onset of convection in a porous medium in the presence of chemical reaction, Phys. Rev. E, 83(4), doi: 10.1103/PhysRevE.83.046312.

Appelo, C., and D. Postma (2005), Geochemistry, Groundwater and Pollution, Second Edition, CRC Press, doi:10.1201/9781439833544.

Backhaus, S., K. Turitsyn, and R. E. Ecke (2011), Convective instability and mass transport of diffusion layers in a Hele-Shaw geometry, Phys. Rev. Lett., 106(10), doi: 10.1103/PhysRevLett.106.104501.

Batchelor, G. K. (1959), Small-scale variation of convected quantities like temperature in turbulent fluid part 1. general discussion and the case of small conductivity, J. Fluid Mech., 5(01), 113-133, doi:10.1017/s002211205900009x.

Bear, J. (1972), Dynamics of fluids in porous media, American Elsevier, New York. 
Bolster, D. (2014), The fluid mechanics of dissolution trapping in geologic storage of $\mathrm{CO}_{2}$, J. Fluid Mech., 740, 14, doi:10.1017/jfm.2013.531.

Cardoso, S. S. S., and J. T. H. Andres (2014), Geochemistry of silicate-rich rocks can curtail spreading of carbon dioxide in subsurface aquifers, Nature Communications, 5, 5743, doi:10.1038/ncomms6743.

Couch, S., R. S. J. Sparks, and M. R. Carroll (2001), Mineral disequilibrium in lavas explained by convective self-mixing in open magma chambers, Nature, 411 (6841), 10371039, doi:10.1038/35082540.

De Simoni, M., J. Carrera, X. Sánchez-Vila, and A. Guadagnini (2005), A procedure for the solution of multicomponent reactive transport problems, Water Resour. Res., 41 (11), W11,410, doi:10.1029/2005WR004056.

deMello, A. J. (2006), Control and detection of chemical reactions in microfluidic systems, Nature, 442 (7101), 394-402, doi:10.1038/nature05062.

Eckert, K., and A. Grahn (1999), Plume and finger regimes driven by an exothermic interfacial reaction, Phys. Rev. Lett., 82, 4436-4439, doi:10.1103/PhysRevLett.82.4436.

Fredd, C. N., and H. S. Fogler (1998), Influence of transport and reaction on wormhole formation in porous media, AIChE J., 44(9), 1933-1949, doi:10.1002/aic.690440902.

Gabrovšek, F., and W. Dreybrodt (2010), Karstification in unconfined limestone aquifers by mixing of phreatic water with surface water from a local input: A model, J. Hydrol, 386(1-4), 130-141, doi:10.1016/j.jhydrol.2010.03.015.

Gérard, T., T. Tóth, P. Grosfils, D. Horváth, A. De Wit, and A. Tóth (2012), Hot spots in density fingering of exothermic autocatalytic chemical fronts, Phys. Rev. E, 86, 016,322, 
doi:10.1103/PhysRevE.86.016322.

Golfier, F., C. Zarcone, B. Bazin, R. Lenormand, D. Lasseux, and M. Quintard (2002), On the ability of a darcy-scale model to capture wormhole formation during the dissolution of a porous medium, J. Fluid Mech., 457, doi:10.1017/S0022112002007735.

Hewitt, D. R., J. A. Neufeld, and J. R. Lister (2013), Convective shutdown in a porous medium at high Rayleigh number, J. Fluid Mech., 719, 551-586, doi: 10.1017/jfm.2013.23.

Hidalgo, J. J., J. Fe, L. Cueto-Felgueroso, and R. Juanes (2012), Scaling of convective mixing in porous media, Phys. Rev. Lett., 109(26), doi:10.1103/PhysRevLett.109.264503.

Hidalgo, J. J., C. W. MacMinn, and R. Juanes (2013), Dynamics of convective dissolution from a migrating current of carbon dioxide, Ad. Water Resour., 62, 511-519, doi:10.1016/j.advwatres.2013.06.013.

Jenny, P., J. S. Lee, D. W. Meyer, and H. A. Tchelepi (2014), Scale analysis of miscible density-driven convection in porous media, J. Fluid Mech., 749, 519-541, doi: 10.1017/jfm.2014.229.

Kechagia, P. E., I. N. Tsimpanogiannis, Y. C. Yortsos, and P. C. Lichtner (2002), On the upscaling of reaction-transport processes in porous media with fast or finite kinetics, Chemical Engineering Science, 57(13), 25652577, doi:10.1016/s0009-2509(02)00124-0.

Kouchi, A., and I. Sunagawa (1983), Mixing basaltic and dacitic magmas by forced convection, Nature, 304(5926), 527-528.

Le Borgne, T., M. Dentz, D. Bolster, J. Carrera, J.-R. de Dreuzy, and P. Davy (2010), Nonfickian mixing: Temporal evolution of the scalar dissipation rate in heterogeneous porous 
media, Ad. Water Resour., 33(12), 1468-1475, doi:10.1016/j.advwatres.2010.08.006.

Le Borgne, T., M. Dentz, and E. Villermaux (2013), Stretching, coalescence, and mixing in porous media, Phys, Rev. Lett., 110 (20), doi:10.1103/physrevlett.110.204501.

Lichtner, P. C. (1985), Continuum model for simultaneous chemical reactions and mass transport in hydrothermal systems, Geochim. Cosmochim. Acta, 49(3), 779-800, doi: 10.1016/0016-7037(85)90172-3.

Lindeberg, E., and D. Wessel-Berg (1997), Vertical convection in an aquifer column under a gas cap of $\mathrm{CO}_{2}$, Energ. Converis. Manage., 38, S229-234, doi:10.1016/s01968904(96)00274-9.

Loodts, V., C. Thomas, L. Rongy, and A. De Wit (2014), Control of convective dissolution by chemical reactions: General classification and application to $\mathrm{CO}_{2}$ dissolution in reactive aqueous solutions, Phys. Rev. Lett., 113, 114,501, doi: 10.1103/PhysRevLett.113.114501.

Neufeld, J. A., M. A. Hesse, A. Riaz, M. A. Hallworth, H. A. Tchelepi, and H. E. Huppert (2010), Convective dissolution of carbon dioxide in saline aquifers, Geophys. Res. Lett., 37(22), L22,404, doi:10.1029/2010GL044728.

Ottino, J. (1989), The Kinematics of Mixing: Stretching, Chaos, and Transport, Cambridge Texts in Applied Mathematics, Cambridge University Press.

Ranz, W. E. (1979), Applications of a stretch model to mixing, diffusion, and reaction in laminar and turbulent flows, AIChE J., 25(1), 41-47, doi:10.1002/aic.690250105.

Riaz, A., M. Hesse, H. A. Tchelepi, and F. M. Orr Jr. (2006), Onset of convection in a gravitationally unstable diffusive boundary layer in porous media, J. Fluid Mech., 548, 
87-111, doi:10.1017/S002211200500749.

Ritchie, L. T., and D. Pritchard (2011), Natural convection and the evolution of a reactive porous medium, J. Fluid Mech., 673, 286317, doi:10.1017/s0022112010006269.

Sanchez-Vila, X., M. Dentz, and L. D. Donado (2007), Transport-controlled reaction rates under local non-equilibrium conditions, Geophys. Res. Lett., 34 (10), doi: 10.1029/2007gl029410.

Schmidt, W. (2006), Turbulence: From tea kettles to exploding stars, Nat. Phys., 2(8), 505-506.

Slim, A. C. (2014), Solutal-convection regimes in a two-dimensional porous medium, J. Fluid Mech., 741, 461491, doi:10.1017/jfm.2013.673.

Slim, A. C., M. M. Bandi, J. C. Miller, and L. Mahadevan (2013), Dissolution-driven convection in a hele-shaw cell, Phys. Fluids, 25 (2), 024,101, doi:10.1063/1.4790511.

Steefel, C. I., and A. C. Lasaga (1990), Evolution of Dissolution Patterns, chap. 17, pp. 212-225, doi:10.1021/bk-1990-0416.ch016.

Szymczak, P., and A. J. C. Ladd (2009), Wormhole formation in dissolving fractures, J. Geophys. Res.-Sol. EA, 114(B6), B06,203, doi:10.1029/2008JB006122.

Szymczak, P., and A. J. C. Ladd (2011), Instabilities in the dissolution of a porous matrix, Geophys. Res. Lett., 38(7), n/a-n/a, doi:10.1029/2011GL046720.

Szymczak, P., and A. J. C. Ladd (2013), Interacting length scales in the reactiveinfiltration instability, Geophys. Res. Lett., 40 (12), 30363041, doi:10.1002/grl.50564.

Tackley, P. J. (2000), Mantle convection and plate tectonics: Toward an integrated physical and chemical theory, Science, 288(5473), 2002-2007, doi: 
10.1126/science.288.5473.2002.

Tél, T., A. de Moura, C. Grebogi, and G. Károlyi (2005), Chemical and biological activity in open flows: A dynamical system approach, Phys. Rep., 413(2-3), 91 - 196, doi: 10.1016/j.physrep.2005.01.005.

Verdon, J., and A. W. Woods (2007), Gravity-driven reacting flows in a confined porous aquifer, J. Fluid Mech., 588, doi:10.1017/s0022112007007069.

Villermaux, E. (2012), Mixing by porous media, C. R. Mecanique, 340(11-12), 933-943, doi:10.1016/j.crme.2012.10.042. 


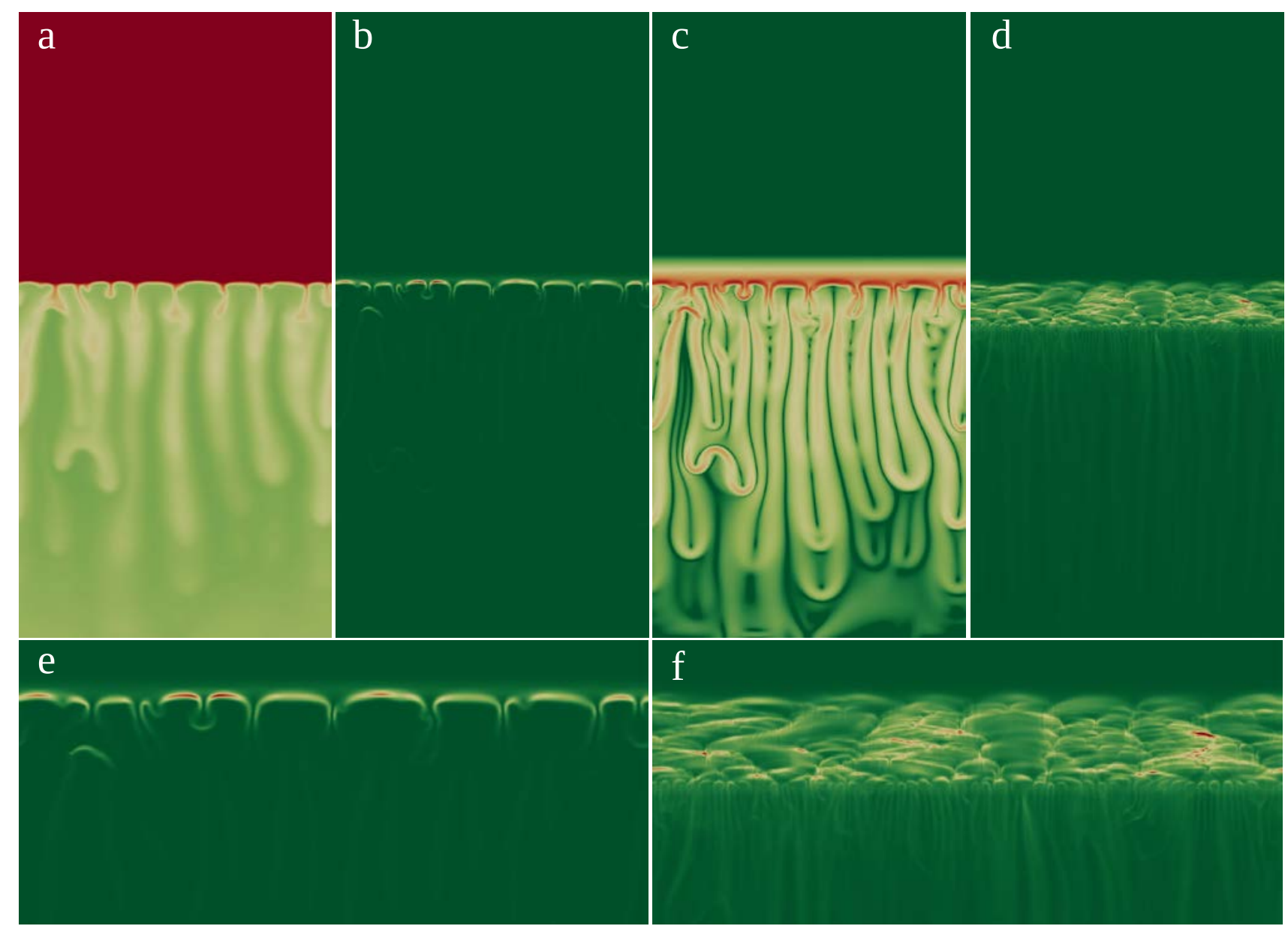

Figure 1. Simulation results for two fluids at close states of equilibrium $\left(U_{T}=10.0\right.$, $\left.U_{B}=0.49\right)$ with a porous matrix similar to a carbonate rock $\left(\mathrm{N}_{\varphi}=0.11\right)$ and $\mathrm{Ra}=10000$, at time $t=15$. (a) The mixing ratio $\alpha$ is characterized by density fingers caused by convective mixing. (b) The dissolution rate $r_{\varphi}$ is concentrated at the interface between the two fluids. (c) The log-dissolution rate shows that reactions are much weaker around the fingers. (d) The resulting pattern of porositz1change reflects the path of the ascending interface. Panels (e) and (f) illustrate the details of the reaction rate (b) and porosity pattern (d) around the fluid interface. See the Supplementary Material for videos [Sup, b]. 

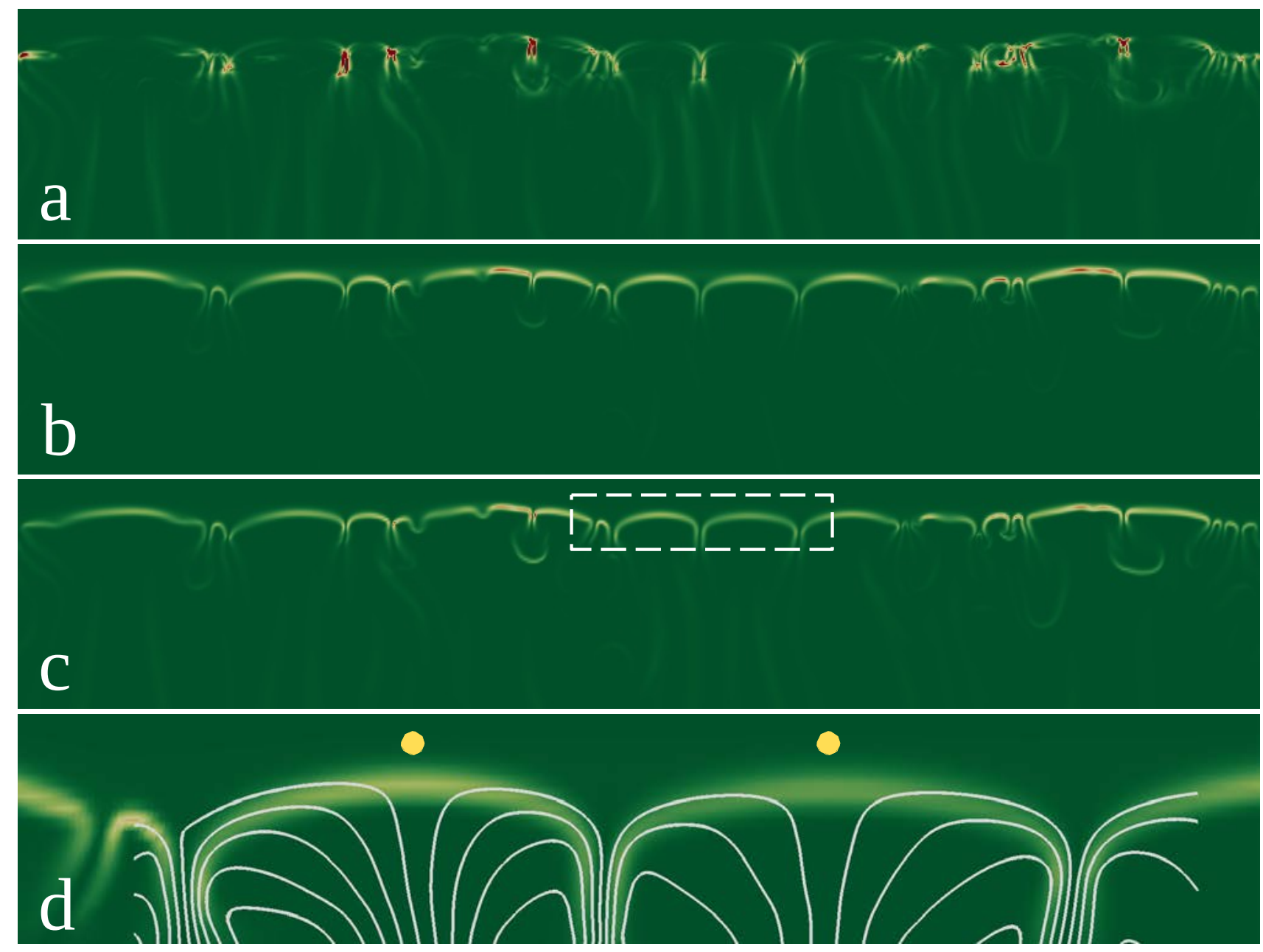

Figure 2. (a) Distribution of the strain rate given by the the determinant of the strain tensor $\left(\nabla \mathbf{q}+\nabla \mathbf{q}^{T}\right) / 2$ [Ottino, 1989], whose maxima are localized at the interface.

The flow stagnation points act as hot spots for mixing and reaction as shown by the dissolution rate (b) and scalar dissipation rate (c). (d) Detail of the mixing hot spots at the stagnation points (yellow dots), and the flow streamlines for the box in (c). 

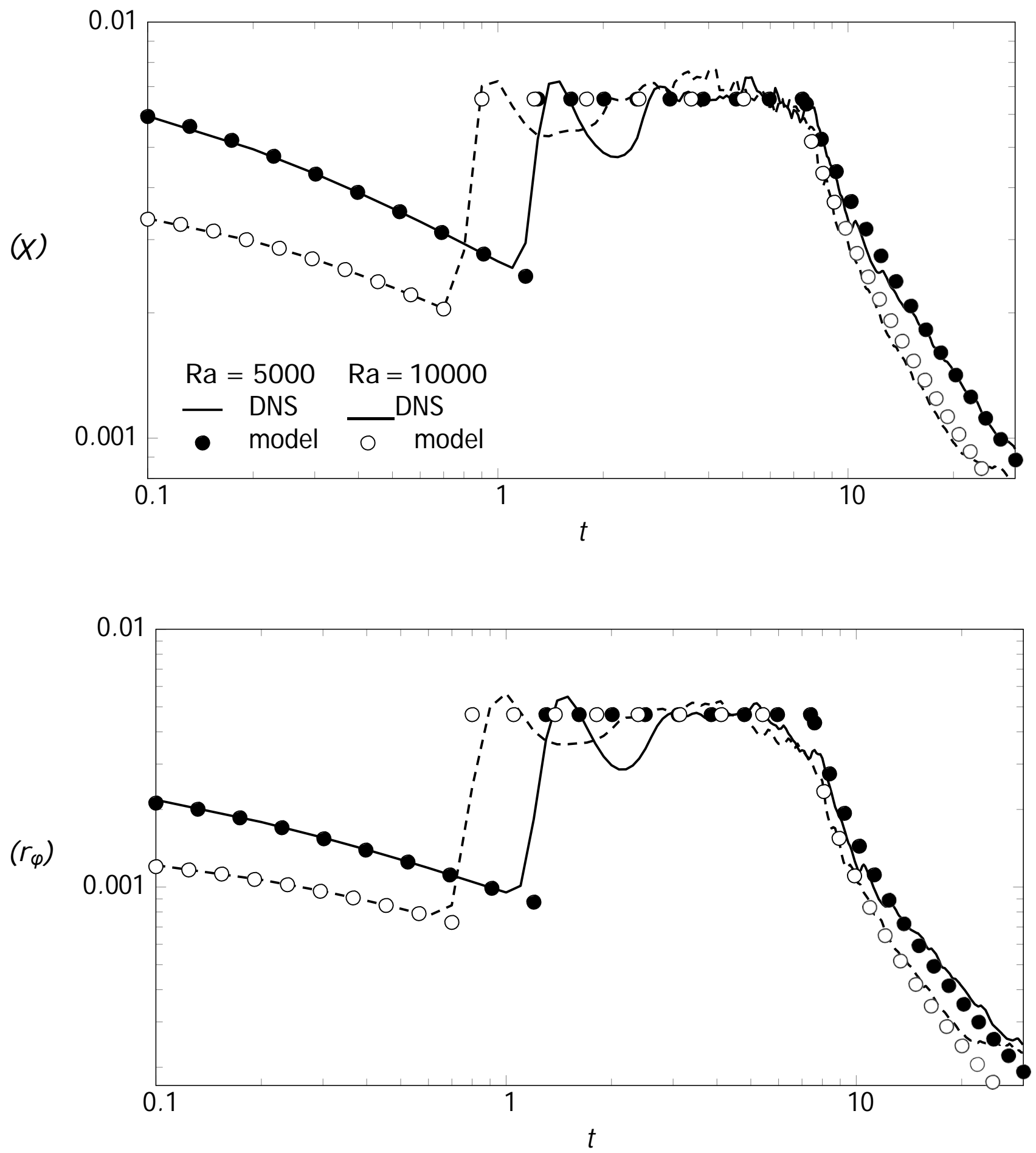

Figure 3. Scalar dissipation rate (top) and reaction rate (bottom) for $\mathrm{Ra}=5000,10000$ $\left(U_{T}=10.0, U_{B}=0.49, \mathrm{~N}_{\varphi}=0.11\right)$. Both magnitudes experience the same three regimes: diffusion dominated $\left(\sim t^{-1 / 2}\right)$; convection domizgted, independent of Ra, and convection shutdown in which reactions attenuate faster than mixing. Lines correspond to the direct numerical simulations and dots to the interface evolution model. 